\title{
Mapping and analyzing process of cloud-based education as a service (CEaaS) model for cloud computing adoption in higher education institutions
}

\begin{abstract}
A newly evolved computing paradigm used to deliver everything-as-a-service (XaaS) for various business organizations is known as Cloud Computing (CC). It is used most especially by educational organizations for educational purposes anywhere and anytime via scalability and an approach that saves cost (pay-per-use). There are a number of problems faced by Higher Education Institutions (HEIs) such as the growing need of infrastructure and IT, providing good and affordable educational services, increasing of participants, increasing education quality provision. Cloud Computing (CC) provides an excellent substitute for HEIs by supporting a reduction in cost, improvement in quality, through this means sustainability in education is obtained by providing good educational services. Recent research patterns on $\mathrm{CC}$ are directly focused on the applications, benefits, costs, technology, and security of CC. However, it was noticed that less empirical evidence and research focus is been given to the adoption of CC at an organization level. A model is proposed with a mapping matrix of its constructs based on the four dominant theories in the field of this adopted technology, the theories are: Technology-organization-environment (TOE) theory, The Fit-Viability Model (FVM), Diffusion of Innovation (DOI) Theory, and Institutional (INT) theory. Implications, potential contributions to research, and suggestions for future study are discussed.
\end{abstract}

Keyword: Cloud computing adoption; Education as a service (EaaS); TOE framework; FVM; DOI; INT theory; Higher education institutions (HEIs) 